\title{
ESTUDIO DE LOS MECANISMOS DE APOPTOSIS Y MITOSIS EN EL GLOBO OCULAR. MODELO EXPERIMENTAL DEL SÍNDROME TÓXICO GESTACIONAL
}

\section{STUDY OF APOPTOSIS AND MITOSIS MECHANISMS IN THE EYE. EXPERIMENTAL MODEL OF GESTATIONAL TOXIC SYNDROME}

\author{
PONS-VÁZQUEZ $\mathrm{S}^{1}$, VILA-BOU V ${ }^{2}$, ZANON-MORENO V${ }^{1}$, IBORRA FJ ${ }^{3}$, GALLEGO-PINAZO R ${ }^{4}$, \\ MELO PM ${ }^{3}$, GARCÍA-MEDINA JJ², PINAZO-DURÁN MD ${ }^{2}$
}

\section{RESUMEN}

Objetivo: Profundizar en los conocimientos de los mecanismos de diferenciación y proliferación celular durante el desarrollo de la retina, estudiando los mecanismos de mitosis y apoptosis en un modelo de exposición prenatal al alcohol en la rata.

Método: Se utilizaron ratas Wistar (200 g peso) y su descendencia, en dos grupos alimentados con dieta líquida: 1) el grupo expuesto al etanol (5\% etanol peso/volumen como 35\% calorías diarias totales) y 2) un grupo control isocalórico (carbohidratos como $35 \%$ calorías diarias totales). Se obtuvieron los globos oculares el día 21 de gestación para incluirlos en parafina y realizar la inmunodetección de células apoptóticas (TUNEL) y mitóticas

\begin{abstract}
Objective: To improve knowledge of the mechanisms of cellular differentiation and proliferation during retinal development, by studying cellular and molecular damage in a rat model of prenatal ethanol exposure.

Methods: Female, juvenile Wistar rats (200g body weight) and their offspring were divided into two groups, which were fed a liquid diet: 1) ethanolexposed group (5\% ethanol weight/vol as $35 \%$ of daily total calories) and 2) isocaloric control group (maltose/dextrin as 35\% of daily total calories). Eyeballs were obtained at 21 days of gestation, embedded in paraffin, and immunodetection procedures performed on apoptotic (TUNEL) and mitotic
\end{abstract}

\footnotetext{
Recibido: 31/5/06. Aceptado: 28/11/07.

1 Licenciado en Biología.

2 Doctor en Medicina.

3 Doctor en Biología.

${ }^{4}$ Licenciado en Medicina. Melo.

Correspondencia:

Sheila Pons Vázquez

Unidad Investigación Oftalmológica Santiago Grisolía

Avenida Gaspar Aguilar 90

46017 Valencia

España

E-mail: pons_she@gva.es
}

Unidad Investigación Oftalmológica Santiago Grisolía. Valencia. Weatherhall Institute of Molecular Biology, Radcliffe Hospital, University of Oxford (UK) e Institute of Molecular and Cell Biology, University of Porto (Portugal).

Este trabajo ha sido subvencionado por un proyecto de investigación FIS-FEDER (P1020191) del Instituto de Salud Carlos III (Investigador Principal Dra. Pinazo-Durán) y beca de formación en investigación oftalmológica asociada a este proyecto otorgada a Sheila Pons Vázquez, y por un proyecto de investigación Fundaçao para a ciencia e tecnologia, Portugal (praxis XXI/BD/3395, praxis P/SAU/12287/1998) (Investigador Principal Prof. Tavares) y beca de formación en investigación oftalmológica asociada a este proyecto otorgada a Pedro M Martins de Almeida 
que se fotografiaron a microscopía confocal, realizando análisis morfológico y morfométrico para estudiar estadísticamente los datos.

Resultados: Las microfotografías revelaron un aumento significativo de perfiles apoptóticos $(\mathrm{p}<0,05)$ y paralelamente un descenso de procesos mitóticos en el grupo expuesto al etanol frente al control. Las células ganglionares y los fotorreceptores presentaron más diferencias en estos dos procesos que el resto de fenotipos celulares retinianos. Los datos obtenidos sugieren anomalías en los procesos de diferenciación y proliferación celular de la retina causados por la exposición al alcohol.

Conclusiones: El abuso de alcohol durante la gestación altera el desarrollo de la retina por inducir anomalías en los procesos mitóticos y apoptóticos. El aumento de apoptosis y disminución de las mitosis pueden deberse a cambios en la expresión de genes reguladores, así como en las vías de señalización de ambos procesos en estadios precoces del desarrollo.

Palabras clave: Alcohol, intoxicación crónica, neurotoxicologia, apoptosis, mitosis. profiles, which were observed and photographed using a confocal microscope.

Results: Analysis of the microphotographs revealed a statistically significant increase of apoptotic profiles and a decrease in mitotic profiles in the ethanol exposed group compared to controls $(\mathrm{p}<0.05)$. Ganglion cells and photoreceptors showed more changes than other retinal cell phenotypes. These findings suggest that abnormalities in the differentiation and proliferation processes of the retina were caused by the alcohol exposure.

Conclusions: Alcohol abuse during pregnancy alters development of the visual system by inducing developmental changes in the mitotic and apoptotic processes of the retina. These latter changes may be the result of changes in the expression of regulatory genes as well as the result of alteration in signalling pathways for both differentiation-proliferation and apoptotic events (Arch Soc Esp Oftalmol 2008; 83: 37-44).

Key words: Ethanol, chronic intoxication, neurotoxicology, apoptosis, mitosis.

\section{INTRODUCCIÓN}

El diagnóstico de los síndromes tóxicos gestacionales (STG) se ha efectuado a lo largo de la historia basándose en antecedentes del abuso del agente tóxico (drogas ilícitas o alcohol) por la mujer gestante. Las descripciones coinciden con la presentación de una «facies» característica y un recién nacido de bajo peso, nacido de madres que han consumido alcohol o drogas. Aunque los efectos nocivos del abuso de alcohol sobre el organismo en desarrollo habían sido sospechados a lo largo de la historia, la tríada patognomónica: malformaciones cráneofaciales, retraso mental y déficit en el crecimiento, fue establecida en 1973 y denominada síndrome alcohólico fetal (SAF) por Jones y Smith (1). A partir de entonces surgieron diversos trabajos epidemiológicos y experimentales que analizaban las consecuencias de la exposición gestacional al alcohol (2-6). El examen oftalmológico de los niños que padecían SAF demostró la disminución significativa de agudeza visual, elevada incidencia de defectos de refracción, estrabismo, anomalías de los segmentos anterior y medio y alteraciones del fondo ocular, cuando los niños con diagnóstico del SAF se compararon con controles de la misma edad y sexo nacidos de madres que no habían ingerido bebidas alcohólicas durante el embarazo (7-10). Sin embargo aún no se han demostrado las bases celulares de los efectos del alcohol y otros agentes teratogénicos, sobre el sistema nervioso. Es bien conocido que durante el desarrollo del sistema visual tanto los procesos de diferenciación y proliferación celular como la muerte programada de los elementos supernumerarios o dañados, conocida como apoptosis, desempeñan una función primordial para la organización citoarquitectural de los tejidos neurales y para el correcto establecimiento de la función visual. Si se analiza el porcentaje de eliminación de las células ganglionares retinianas de la rata, aproximadamente un $90 \%$ de éstas mueren durante la primera semana tras el nacimiento, de manera que aquellas que consiguen sobrevivir realizan las sinapsis con sus elementos diana que a su vez aportarán los factores tróficos necesarios para inhibir las vías de señalización de la apoptosis, permitiendo que sobrevivan.

Puesto que se ha descrito con anterioridad que el abuso crónico de alcohol interfiere con el metabolismo y la señalización de diversos procesos esen- 
ciales para el mantenimiento de la salud (11) y se han definido las vías de señalización de la apoptosis en relación a diversas patologías oftalmológicas (12), en el presente trabajo postulamos que el alcohol, como agente teratógeno puede inducir daño celular irreversible en estadios precoces del desarrollo de la retina, compatibles con las características oftalmológicas que presentan los niños con el síndrome tóxico gestacional, realizando los experimentos para evaluar los procesos de diferenciaciónproliferación celular y apoptosis en un modelo de exposición pre- y postnatal en la rata.

\section{SUJETOS, MATERIAL Y MÉTODOS}

\section{Animales: grupos, dietas y obtención del tejido}

El modelo experimental se llevó a cabo en la rata Wistar (5,13-16) y se ajustó a los requerimientos de la CE para este tipo de estudios de investigación animal. ocho ratas hembras de la raza Wistar y en edad juvenil (200 g de peso) se aclimataron a las condiciones estándar de laboratorio durante una semana y fueron divididas en dos grupos: 1) cuatro ratas que recibieron una dieta líquida en las que el etanol (5\% peso/volumen) aportó el 35\% de las calorías diarias (GETOH) 2) cuatro ratas que fueron alimentadas con dieta liquida conteniendo maltosa-dextrina en la misma proporción que el etanol en el grupo anterior y que se consideró como grupo control nutricional paralelamente alimentado (GCN). Las ratas se mantuvieron con esta alimentación durante 6 semanas, anotando diariamente su ingesta y peso corporal. La alcoholemia se midió en la sangre obtenida de la cola, antes y durante la gestación, y se analizó por cromatografía de gases (5). Luego se aparearon y se llevó un control estricto durante toda la gestación. El día 21 de gestación (G21) dos ratas de cada grupo fueron sacrificadas por decapitación para obtener los fetos. Éstos fueron examinados, pesados, determinada su alcoholemia y clasificados de acuerdo a sus características y edad. cuatro fetos de cada rata, de ambos grupos, fueron decapitados para obtener los globos oculares y nervios ópticos. Unos se destinaron a mediciones para obtener diversos parámetros del desarrollo mediante microscopía óptica y otros se procesaron para técnicas de inmunocitoquímica a microscopía confocal.

Los ojos de los fetos fueron fijados con paraformaldehído $4 \%$ durante toda la noche. Después se lavaron con PBS para incluir las muestras en parafina y realizar los cortes y así observar las características histológicas que no se podían apreciar de forma óptima cuando se microseccionaba directamente con criostato.

Para la inclusión en parafina se utilizó el procedimiento estándar, incluyendo las muestras en parafina y realizando los cortes y desparafinando los mismos antes de empezar con las técnicas inmunohistoquímicas.

\section{Protocolo para la identificación y recuento de células apoptóticas}

Protocolo de la técnica TUNEL [terminal dUTP nick end labeling (TUNEL)]. Las secciones transversales del espesor de la retina se pretrataron con 0,02\% Tritón X-100 en PBS-tween 20 (0,01\%) durante 30 minutos y se preincubaron con tampón de reacción TdT durante 10 minutos. A continuación se incubaron, dos horas en una cámara húmeda a $40-45^{\circ} \mathrm{C}$, con $\mathrm{TdT}$ reaction mixture que está formado por: enzima TdT 4 $\mu$, tampón TdT $992 \mu l$, biotina 16 dUTP $4 \mu 1$. Rápidamente se lavó en el mismo tampón que en la preincubación durante 10 min. Para parar la reacción se lavó tres veces durante dos minutos con PBS-Tween 20 (0,01\%). Seguidamente se incubó con FITC-Avidin D de Vector Labs (1:50) en PBS durante 30 minutos a temperatura ambiente. Después se lavó tres veces durante dos minutos con PBS-Tween 20 (0,01\%). Se realizó el contraste con PI Counterstain Solution" por 30 min, y se volvió a lavar con PBS durante cinco minutos. Las secciones fueron montadas para posterior visualización al microscopio confocal Olympus BX 51 (Olympus, Tokio, Japón).

\section{Protocolo para la identificación y recuento de células mitóticas}

Las secciones transversales de la retina de las ratas se sumergieron en Tritón $1 \%$ diez minutos, y se lavaron con PBS para sumergir de nuevo con PBBSA (PBS + Bovine Serum Albumin) durante 30 minutos. Se incubaron con el anticuerpo primario Proliferating Cell Nuclear Antigen (PCNA) $2,5 \%$ en BSA durante una hora, y se lavaron con PBS tres veces de cinco minutos. A continuación se añadió el anticuerpo secundario Cy 3 (Jackson 
Inmuno Research) al 0,5\% en PBBSA durante una hora, y se lavó con PBS tres veces durante cinco minutos. Seguidamente se añadió TOPRO 3 iodide (Molecular Probes T3605) 0,02\% en PBS durante cinco minutos y se lavó con PBS cinco minutos. A continuación las secciones fueron montadas para posterior visualización al microscopio confocal Olympus BX 51.

\section{Análisis estadístico}

Para validar las características del modelo experimental de exposición prenatal al alcohol, se determinó la concentración de alcohol en la sangre de la cola tanto a las madres gestantes (primera, segunda y tercera semana de la gestación) como a los fetos, se determinó la ingesta de comida y el aumento de peso corporal y se registraron los datos analizando la media y desviación estándar, expresadas en gramos, conforme a nuestros estudios previos (15-17). Se pesaron los globos oculares de los fetos, inmediatamente tras la extracción, utilizando una balanza de precisión Metzler y se anotaron los datos respectivos para analizar la media y desviación estándar, expresadas en miligramos. Las micrografias de los nervios ópticos se analizaron mediante los datos correspondientes a los diámetros mayor y menor de la sección transversal y se halló el área de la sección aproximando al área de una circunferencia (que se asemeja a la apariencia morfológica del nervio óptico en el animal inmaduro) siguiendo nuestros estudios anteriores (17-20), registrando los datos para analizarlos hallando la media y desviación estándar de la muestra, expresados en micras cuadradas. Las micrografias de las retinas se examinaron y se hallaron las medias y desviación estándar de las medidas de la sección transversal incluyendo todas las células del espesor total del tejido, siguiendo nuestros trabajos publicados con anterioridad (18-20).

Todos estos datos se han expresado como la media y desviación estándar de 4-6 microfotografias por rata.

En cuanto a la inmunodetección de las células apoptóticas o mitóticas, según cada técnica empleada se contabilizaron las células de tinción positiva para cada caso respecto el área medida en $\mu \mathrm{m}^{2}$ y se expresaron como densidad de células apoptóticas y/o densidad de células mitóticas, utilizando para ello 4-6 micrografías por cada animal y tejido analizado.
Todos los datos fueron registrados en una hoja diseñada para este fin mediante el programa Excel para Windows (Excel for Windows, Illinois, USA) y fueron analizados estadísticamente mediante el programa SPSS 11.0 (SPSS for Windows, SPSS Inc, Chicago, USA). Las diferencias entre grupos fueron analizadas mediante el test «t» de Student y alternativamente con el test de Kolmogorov-Smirnov.

\section{RESULTADOS}

El modelo experimental en la rata demostró la disminución del peso y tamaño de los animales expuestos al etanol frente a los controles durante el período de desarrollo prenatal, así como del tamaño y peso del globo ocular durante la fase final de la gestación (en G21: 18 DE 4 vs 10 DE $2 \mathrm{mg}$ ). De forma similar, el espesor de la retina prenatal fue significativamente menor en los animales expuestos al tóxico (en G21:128 DE 23 vs $81 \mathrm{DE} 14 \mu \mathrm{m}$ ), así como el área de la sección transversal del nervio óptico (en G21:50.000 DE 8.000 vs 31.000 DE $9.000 \mu \mathrm{m}^{2}$ ) fue significativamente inferior en el grupo expuesto al alcohol que en el grupo control.

Por otra parte, la realización de las inmunodetecciones en las secciones transversales de la retina tratadas con la técnica del TUNEL y el análisis pormenorizado de las fotos de la retina en fetos de rata de 21 días confirma la utilidad de la técnica para estudiar los fenómenos de apoptosis en la retina en el periodo prenatal, lo que puede ser tenido en cuenta tanto para los estudios del desarrollo retiniano como para los trabajos relacionados con la neurotoxicología del desarrollo.

Los perfiles de células en proceso de apoptosis quedan reflejados por la inmunotinción en verde. Los datos de nuestro experimento muestran un aumento significativo estadísticamente de las células apoptóticas respecto al área $\left(\mu \mathrm{m}^{2}\right)$ en el grupo expuesto al etanol, frente a los controles, conforme se puede observar en la figura 1 y 3 .

Cuando se analizaron las inmunodetecciones en el microscopio confocal correspondientes a las secciones transversales de la retina con la técnica de identificación de perfiles mitóticos se observa un descenso notable de los procesos mitóticos en el grupo expuesto al etanol, frente al grupo control, aunque no llega a ser significativo estadísticamente conforme se puede observar en la figura 2 y 4 . 

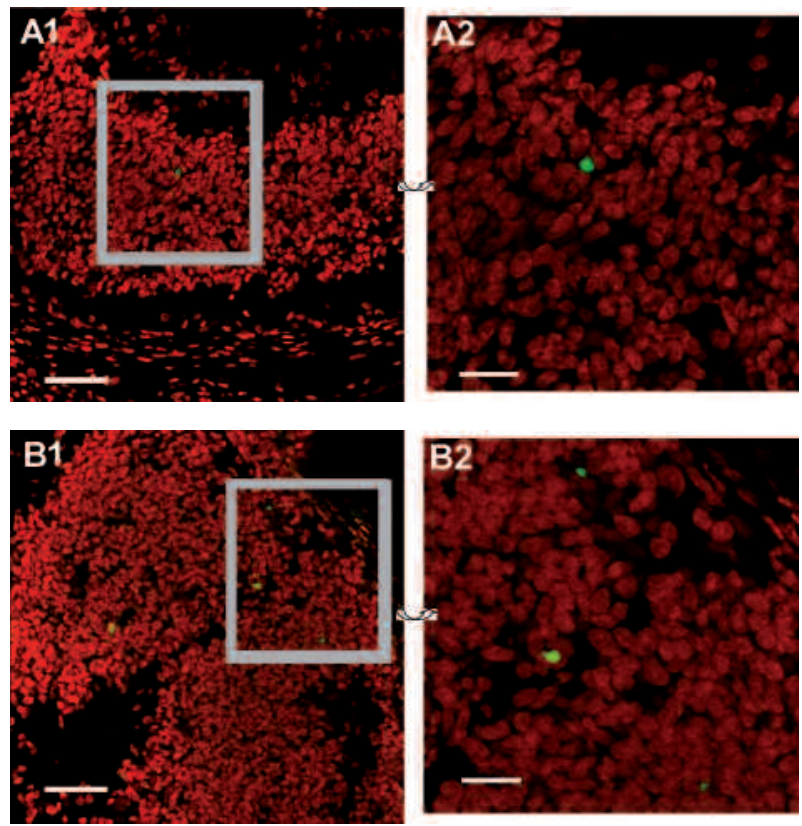

Fig. 1: Microfotografía Confocal. Sección transversal de la retina de la rata. Técnica de TUNEL. Perfil apoptótico de retina de feto de rata de 21 días expuesta prenatalmente al alcohol. A: Grupo Control, B: Grupo

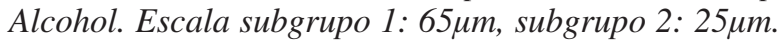

\section{DISCUSIÓN}

Las mujeres que ingieren bebidas alcohólicas de forma abusiva o presentan dependencia de psicotropos durante el embarazo corren el riesgo de tener hijos que muestren un patrón de malformaciones que pueden oscilar desde anomalías menores (reco-

\section{APOPTOSIS}

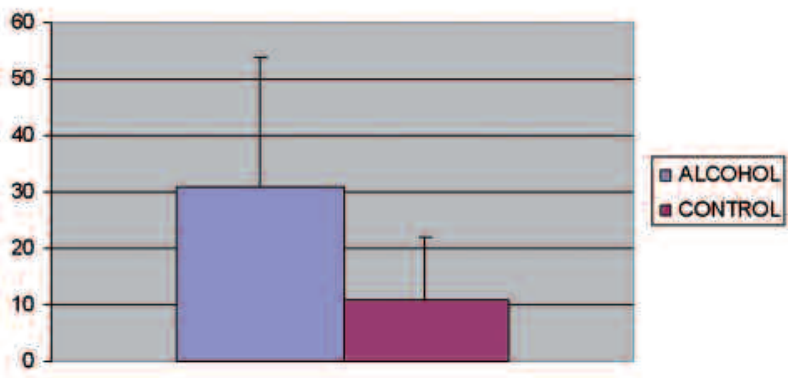

Fig. 3: Después de realizar el estudio estadístico de la $t$-Student se obtuvo un p-valor $<0,05$, con lo que se demuestra en nuestro estudio que existe un mayor número de células apoptóticas en el grupo alcohol que en el control.
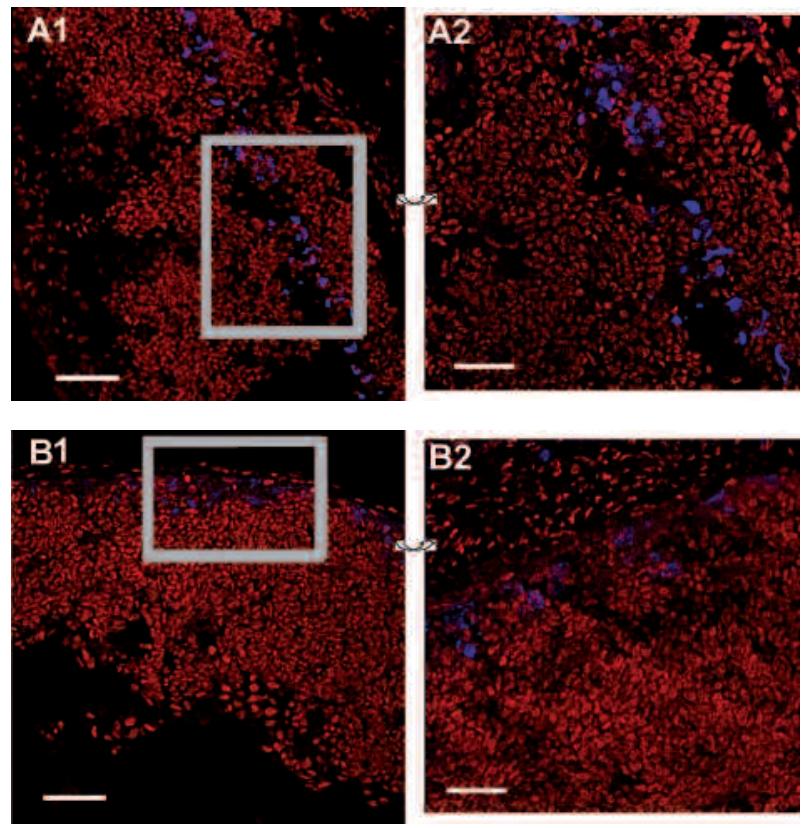

Fig. 2: Microfotografía Confocal. Sección transversal de la retina de la rata. Inmunotinción. Perfil mitótico de retina de feto de rata de 21 días expuesta prenatalmente al alcohol. A: Grupo Control, B: Grupo Alcohol. Escala subgrupo 1: $70 \mu \mathrm{m}$, subgrupo 2: $35 \mu \mathrm{m}$.

nocidas mundialmente como defectos de recién nacido asociados al abuso de alcohol y drogas «drug and alcohol related birth defects») hasta manifestaciones multiorgánicas severas, las cuales en su máxima expresión constituyen el síndrome alcohólico fetal (1-4) y el síndrome fetal de la cocaína y metamfetamina $(21,22)$. La frecuencia estima-

\section{MITosis}

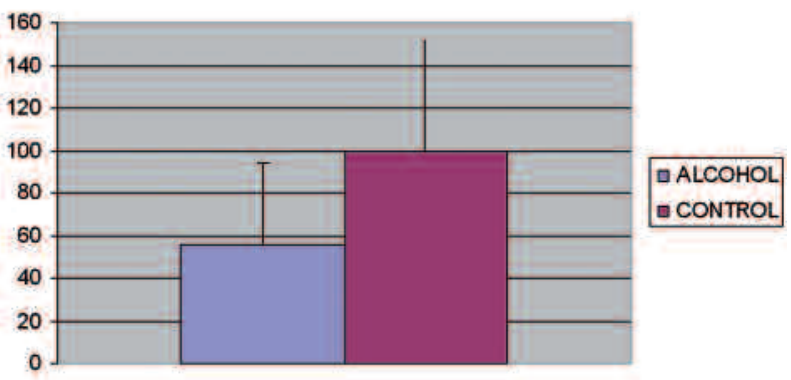

Fig. 4: Después de realizar el estudio estadístico de la $t$-Student se obtuvo un p-valor $>0,05$, por lo que no hay una diferencia significativa estadísticamente. Pero se observa una diferencia notable entre el grupo alcohol y control. 
da de estos síndromes tóxicos es similar en los países industrializados, oscilando entre 1 a 2/1.000 niños nacidos vivos para el alcohol $(23,24)$. Sin embargo, la falta de reconocimiento de los signos patognomónicos de estos síndromes, unido a la ausencia de trabajos epidemiológicos en nuestro país, nos permite incidir en el tema, puesto que no creemos que estos datos sean muy diferentes en el resto de países de Europa (25). Sin embargo, el desconocimiento de los cuadros típicos hace que muchos niños sean diagnosticados como posible «sufrimiento fetal» y/o malformación congénita de origen desconocido y frecuentemente como niños difíciles o hiperactivos por educadores y psicólogos.

Por un lado, teniendo en cuenta la especial vulnerabilidad del sistema visual durante el desarrollo, la exposición al tóxico durante la embriogénesis y período perinatal explicaría «per sé» la alteración en los patrones de morfogénesis ocular. Durante las primeras 8 semanas tras la concepción, todos los órganos y sistemas vitales han comenzado a desarrollarse, de manera que la placa neural comienza a formarse en la tercera semana y el macizo facial y el primordio ocular aparecen alrededor de la cuarta semana. El desarrollo del embrión continúa y existe otro período vital para el desarrollo del SNC y estructuras relacionadas con él, y que abarca desde el tercer trimestre de gestación hasta el nacimiento y período perinatal en el cual se desarrollan las células neuroglíales y se produce la mielinización axonal. Basándonos en estos hechos, podríamos especular que los efectos teratogénicos del alcohol y de otros tóxicos similares, comienzan probablemente durante el período embrionario precoz y continúan a través de toda la gestación, lo cual hemos podido comprobar con el modelo experimental de SAF en la rata, donde hemos constatado la disminución en el peso y tamaño del animal, así como la disminución en tamaño y peso del globo ocular durante todo el desarrollo, en los ojos expuestos pre y postnatalmente al etanol, resultados que coinciden con otros previos de nuestro grupo de investigación en neurotoxicología del sistema visual (15-22).

La formación del globo ocular requiere una serie de hechos con la interacción de diversos tejidos. Los estadios iniciales implican una muerte celular extensa asociada al propio proceso de morfogénesis. Un poco mas tarde, la supresión de la muerte celular programada es fundamental para la diferenciación del tejido en el adulto. Hay estudios que describen que la supresión de la apoptosis en célu- las del cristalino por factores de crecimiento (FGF) es uno de los principales componentes de sus acciones durante el desarrollo del cristalino (26). La apoptosis inducida es esencial en determinados momentos del desarrollo, por ejemplo durante la reabsorción de la vascularización primitiva. Sin embargo, la aparición de mayor densidad de perfiles apoptóticos en la retina del animal expuesto al etanol frente al control, demuestra que existe un aumento de la muerte celular programada inducida por la exposición al tóxico. Estos hechos, junto a la disminución de los perfiles mitóticos, demuestran una inhibición de la proliferación celular junto al aumento de la muerte de las células retinianas, lo cual coincidiría con las descripciones de un nervio óptico hipoplásico, similar al descrito por nosotros con los parámetros morfológicos y morfométricos utilizados con el modelo experimental que venimos utilizando para nuestros estudios (17-20).

En resumen, la inducción de apoptosis y la disminución de la mitosis subsecuente a la exposición prenatal al alcohol en periodos críticos del desarrollo podría explicar la reducción del tamaño de la retina y nervio óptico y la disminución de la densidad celular que acontece en la hipoplasia del nervio óptico (20), patognomónica de los niños nacidos de madres alcohólicas.

Con este y otros trabajos similares demostramos que ningún período de la gestación escapa a la acción del alcohol, por lo que recomendamos que las madres gestantes no consuman bebidas alcohólicas (tanto las de menor o mayor contenido de alcohol) durante todo el periodo gestacional con el fin de salvaguardar el desarrollo del sistema visual de sus hijos. A raíz de nuestras investigaciones nos permitiríamos aconsejar a las autoridades sanitarias incluir las advertencias pertinentes a este respecto en las bebidas alcohólicas y en los refrescos con contenido alcohólico, tal y como ocurre en otros países occidentales.

\section{BIBLIOGRAFÍA}

1. Jones KL, Smith DW, Ulleland CN, Streissguth AP. Pattern of malformation in offspring of chronic alcoholic mothers. Lancet 1973; 9: 1267-1271.

2. Majewski F, Bierich JR, Loser H, Michaelis R, Lieber B, Bettecken F. Clinical aspects of pathogenesis of alcohol embryopathy. MMW Med Wochenschr 1976; 118: 16351642.

3. Clarren SK, Smith DW. The fetal alcohol syndrome. $N$ Engl J Med 1978; 298: 1063-1067. 
4. Rosset HL. A clinical perspective of the Fetal Alcohol Syndrome. Alcohol Clin Exp Res 1980; 4: 119-122.

5. Sanchis R, Guerri $C$. Alcohol metabolizing enzymes in the placenta and fetal liver: effect of chronic ethanol intake. Alcohol Clin Exp Res 1986; 10: 39-44.

6. Sheller B, Clarren SK, Astley SJ, Sampson PD. Morphometric analysis of Macaca nemestrina exposed to ethanol during gestation. Teratology 1988; 38: 411-417.

7. Miller M, Israel J, Cuttone J. Fetal alcohol syndrome. J Pediatr Ophthalmol Strabismus 1981; 18: 6-15.

8. Stromland K. Ocular abnormalities in fetal alcohol syndrome. Acta Ophthalmol Suppl 1985; 63: 171: 1-50.

9. Chan T, Bowel R, O'Keefe M, Lanigan B. Ocular manifestations in fetal alcohol syndrome. Br J Ophthalmol 1991; 75: 524-526.

10. Hinzpeter EN, Renz, S, Loser H. Eye manifestations of fetal alcohol syndromealkoholembryopathie. Klin Monats Augenheilk 1992; 200: 33-38.

11. Wang XD. Chronic alcohol intake interferes with retinoid metabolism and signaling. Nutr Rev 1999; 57: 51-59.

12. García M, Vecino E. Vías de señalización intracelular que conducen a la apoptosis de las células de la retina. Arch Soc Esp Oftalmol 2003; 78: 351-364.

13. Stromland $K$. Contribution of ocular examination to the diagnosis of fetal alcohol syndrome in mentally retarded children. J Ment Defic Res 1990; 34: 429-435.

14. Renau-Piqueras J, Zaragoza R, De Paz P, Baguena-Cerverella $R$, Megias L, Guerri C. Effects of prolonged ethanol exposure on the glial fibrillary acidic protein-containing intermediate filaments of astrocytes in primary culture: a quantitative immunofluorescence and immunogold electron microscopic study. J Histochem Cytochem 1989; 37: 229-240.

15. Pinazo-Duran MD. Efecto de la exposición pre- y postnatal al alcohol sobre el desarrollo del nervio óptico, en la rata. Thesis Doctoralis. Universidad de Valencia. 1991. pp 247.
16. Pinazo-Duran MD, Renau-Piqueras J, Guerri C. Efectos de la exposición intrauterina y postnatal al alcohol sobre el desarrollo del nervio óptico en la rata. Arch Soc Esp Oftalmol 1992; 63: 225-232.

17. Pinazo-Duran MD, Renau-Piqueras J, Guerri C. Developmental changes in the optic nerve related to ethanol consumption in pregnant rats: analysis of the ethanolexposed optic nerve. Teratology 1993; 48: 305-322.

18. Stromland K, Pinazo-Duran MD. Optic nerve hipoplasia: comparative effects in children and rats exposed to alcohol during pregnancy. Teratology 1994; 50:100-111.

19. Pinazo-Duran MD, Renau-Piqueras J, Guerri C, Stromland $K$. Optic nerve hypoplasia in fetal alcohol syndrome: an update. Eur J Ophthalmol 1997; 7: 262-270.

20. Stromland K, Pinazo-Duran MD. Ophthalmic involvement in the fetal alcohol syndrome: clinical and animal model studies. Alcohol Alcohol 2002; 37: 2-8.

21. Melo P, Moreno VZ, Vázquez SP, Pinazo-Durán MD, Tavares MA. Myelination changes in the rat optic nerve after prenatal exposure to methamphetamine. Brain Res 2006; 1106: 21-29.

22. Melo P, Rodrigues LG, Pinazo-Durán MD, Tavares MA. Methamphetamine and lipid peroxidation in the rat retina. Birth Defects Res A Clin Mol Teratol 2005; 73: 455-460.

23. Abel EL, Sokol RJ. Incidence of fetal alcohol syndrome and economic impact of FAS-related anomalies. Drug Alcohol Depend 1987; 19: 51-70.

24. Strömland K, Hellstrom A. Fetal alcohol syndrome-an ophthalmological and socioeducational prospective study. Pediatrics 1996; 97: 845-850.

25. Stromland K, Chen Y, Norberg T, Wennerstrom K, Michael $G$. Reference values of facial features in scandinavian children measured with a range-camera technique. Scand J Plast Reconstr Hand Surg 1999; 33: 59-65.

26. Lang RA. Apoptosis in mammalian eye development. lens morphogenesis, vascular regression and immune privilege. Cell Death Differ 1997; 4: 12-20. 